\title{
Variational Quantum Simulation of General Processes
}

\author{
Suguru Endo®, ${ }^{1,4, *}$ Jinzhao Sun, ${ }^{2}$ Ying Li, ${ }^{3}$ Simon C. Benjamin, ${ }^{1}$ and Xiao Yuan $\oplus^{1, \dagger}$ \\ ${ }^{1}$ Department of Materials, University of Oxford, Parks Road, Oxford OX1 3PH, United Kingdom \\ ${ }^{2}$ Clarendon Laboratory, University of Oxford, Parks Road, Oxford OX1 3PU, United Kingdom \\ ${ }^{3}$ Graduate School of China Academy of Engineering Physics, Beijing 100193, China \\ ${ }^{4}$ NTT Secure Platform Laboratories, NTT Corporation, Musashino 180-8585, Japan
}

(Received 14 June 2019; revised 11 May 2020; accepted 2 June 2020; published 29 June 2020)

\begin{abstract}
Variational quantum algorithms have been proposed to solve static and dynamic problems of closed many-body quantum systems. Here we investigate variational quantum simulation of three general types of tasks - generalized time evolution with a non-Hermitian Hamiltonian, linear algebra problems, and open quantum system dynamics. The algorithm for generalized time evolution provides a unified framework for variational quantum simulation. In particular, we show its application in solving linear systems of equations and matrix-vector multiplications by converting these algebraic problems into generalized time evolution. Meanwhile, assuming a tensor product structure of the matrices, we also propose another variational approach for these two tasks by combining variational real and imaginary time evolution. Finally, we introduce variational quantum simulation for open system dynamics. We variationally implement the stochastic Schrödinger equation, which consists of dissipative evolution and stochastic jump processes. We numerically test the algorithm with a 6-qubit $2 \mathrm{D}$ transverse field Ising model under dissipation.
\end{abstract}

DOI: 10.1103/PhysRevLett.125.010501

Introduction.-The variational method is a powerful classical tool for simulating many-body quantum systems [1-5]. The core idea is based on the intuition that physical states with low energy belong to a small manifold of the whole Hilbert space. As quantum circuits can efficiently prepare states that may not be efficiently represented classically, the variational method has been recently generalized to the quantum regime with trial states efficiently prepared by a quantum circuit and information extracted from a coherent measurement of the state [6-26]. The trial state in variational quantum algorithms can be prepared with shallow quantum circuits [27-30], which is robust to a certain amount of device noise and is compatible with nearterm noisy intermediate scale quantum (NISQ) hardware [31]. Variational quantum algorithms can be utilized for efficiently finding energy spectra [7-12,19,21-25,32] and simulating real time Schrödinger evolution $[13,33]$ of closed systems. Although quantum circuits are unitary operations, the variational algorithm is not limited to energy minimization and unitary processes and it can be used to simulate dissipative imaginary time evolution that cannot be straightforwardly mapped to unitary gates [20,34].

In this Letter, we study the capability of variational quantum algorithms and show that they are not limited to

Published by the American Physical Society under the terms of the Creative Commons Attribution 4.0 International license. Further distribution of this work must maintain attribution to the author(s) and the published article's title, journal citation, and DOI. these applications. First, we introduce a variational quantum algorithm for simulating the generalized time evolution defined in Eq. (1) below. Our algorithm can be regarded as a unified framework, which incorporates the special cases of real and imaginary time evolutions [13,20,34], non-Hermitian quantum mechanics [35-37] that describes nonequilibrium processes [38], parity-time symmetric Hamiltonians [39-41], open quantum systems [42], general first-order differential equations, etc.

Next we apply the variational method for solving linear algebra problems, such as linear systems of equations and matrix-vector multiplications, important tasks in machine learning and optimization [43,44]. Many algorithms have been developed for linear systems of equations with universal quantum computers [45-52], which have profound applications in quantum machine learning [53-57]. However, they generally require deep circuits that rely on fault tolerant quantum computers. In this Letter, we introduce two types of variational quantum algorithms for the two linear algebra problems. For the first type, we consider general sparse matrices and show how solutions of the problems can be converted into generalized time evolutions, which can be variationally simulated. For the second type, we consider special matrices that are products of small matrices acting on a constant number of qubits, and use variational real and imaginary time evolution to find solutions.

Finally, we combine the developed variational algorithms to simulate the evolution of open quantum systems [58-60]. Simulating the evolution of general open quantum systems is of great importance for understanding any 
quantum system that interacts with an environment. Existing quantum algorithms [61-66] for simulating open quantum systems generally require deep quantum circuits. In this Letter, we consider the description of open system dynamics via the stochastic Schrödinger equation, whose evolution can be regarded as an average of wave functions that undergo a continuous measurement induced from the environment $[60,67]$. The evolution of each wave function is composed of two processes that can both be simulated with variational algorithms: It may continuously evolve under the generalized time evolution with the system Hamiltonian and the damping effect due to continuous measurement; alternatively, the state discontinuously jumps according to the measurement results. The continuous process can be described by the generalized time evolution, and the jump process is a matrix-vector multiplication process. Therefore, our algorithm is compatible with shallow circuits and NISQ hardware.

Generalized time evolution.-We first consider variational quantum simulation of generalized time evolution:

$$
B(t) \frac{d}{d t}|v(t)\rangle=|d v(t)\rangle .
$$

Here $|d v(t)\rangle=\sum_{j} A_{j}(t)\left|v_{j}^{\prime}(t)\right\rangle, A_{j}(t)$, and $B(t)$ are general time dependent sparse (non-Hermitian) operators, $|v(t)\rangle$ is the system state, and each of $\left|v_{j}^{\prime}(t)\right\rangle$ can be either $|v(t)\rangle$ or any known state that can be efficiently prepared with a quantum circuit. The states $|v(t)\rangle$ and $\left|v_{j}^{\prime}(t)\right\rangle$ can be (un)normalized states $|v(t)\rangle=\alpha(t)|\psi(t)\rangle,\left|v_{j}^{\prime}(t)\right\rangle=\alpha_{j}^{\prime}(t)\left|\psi_{j}^{\prime}(t)\right\rangle$, with normalization factors $\alpha(t)$ and $\alpha_{j}^{\prime}(t)$, respectively. In practice, we assume that $A_{j}(t)[B(t)]$ can be decomposed as a linear combination of Pauli operators $A_{j}(t)=\sum_{i} \lambda_{i}^{j}(t) \sigma_{i}$ with complex coefficients $\lambda_{i}$ and a polynomial (with respect to the system size) number of tensor products of Pauli matrices $\sigma_{i}=\otimes i_{i_{k}} \sigma_{i_{k}}$, with $i_{k}$ denoting the $i_{k}$ th qubit.

In variational quantum simulation, instead of directly simulating the dynamics, we assume that the state can be represented by parametrized quantum states $|v(\vec{\theta}(t))\rangle=$ $\alpha\left(\vec{\theta}_{0}(t)\right)\left|\varphi\left(\vec{\theta}_{1}(t)\right)\right\rangle$ with $\vec{\theta}:=\left(\vec{\theta}_{0}, \vec{\theta}_{1}\right)$. Then we project the original evolution to the evolution of the parameters via McLachlan's principle [68],

$$
\min \| B(t) \frac{d}{d t}|v(\vec{\theta}(t))\rangle-\sum_{j} A_{j}(t)\left|v_{j}^{\prime}(t)\right\rangle \|,
$$

where $\||\psi\rangle \|=\sqrt{\langle\psi \mid \psi\rangle}$ and the minimization is over the derivative of the parameters. By minimizing the distance between the true evolution and the evolution of the parametrized state, we find the equation of parameters as

$$
\sum_{j} \tilde{M}_{k, j} \dot{\theta}_{j}=\tilde{V}_{k}
$$

where $\dot{\theta}_{j}=d \theta_{j} / d t$ and the coefficients are linear sums of state overlaps that can be efficiently measured with quantum circuits [69]. We specify the detailed derivation, expression of the coefficients, the quantum circuits, and a detailed resource estimation of the algorithm in Supplemental Material [70]. Here we consider two examples with $B(t)=1$ and $|d v(t)\rangle=-i H|v(t)\rangle$ or $|d v(t)\rangle=$ $-[H-\langle v(t)|H| v(t)\rangle]|v(t)\rangle$, corresponding to real and imaginary time evolution [13,20,34], respectively. Compared to previous works studying real and imaginary time dynamics [13,20,34], our algorithm considers a much more general setting of first-order differential equations. Therefore, it not only unifies previous results in the general setting, but provides the basis for solving general problems, as we discuss below.

Variational algorithms for linear algebra.-Now consider linear algebra problems of solving linear systems of equations and matrix-vector multiplications. For a sparse square matrix $\mathcal{M}$ and a state vector $\left|v_{0}\right\rangle$, we aim to find

$$
\left|v_{\mathcal{M}^{-1}}\right\rangle=\mathcal{M}^{-1}\left|v_{0}\right\rangle \quad \text { or } \quad\left|v_{\mathcal{M}}\right\rangle=\mathcal{M}\left|v_{0}\right\rangle .
$$

We introduce two types of algorithms where the first type is more general and the second type is more efficient with assumptions of the matrix. Here we take matrix multiplication as an example and the derivation works similarly for linear equations, which can also be found in Supplemental Material [70].

For the first type, the algorithm is based on converting the static algebraic problem into a dynamical process, evolving the initial vector $\left|v_{0}\right\rangle$ to the target state $\left|v_{\mathcal{M}}\right\rangle$. A natural evolution path is via a linear extrapolation between $\left|v_{0}\right\rangle$ and $\left|v_{\mathcal{M}}\right\rangle$ as $|v(t)\rangle=E(t)\left|v_{0}\right\rangle$ with $E(t)=$ $(t / T) \mathcal{M}+(1-t / T) I,|v(0)\rangle=\left|v_{0}\right\rangle$, and $|v(T)\rangle=\left|v_{\mathcal{M}}\right\rangle$. Different evolution paths can also be considered. For example, in the conventional Hamiltonian simulation scenario [71-75], we have $\mathcal{M}=e^{-i H T}$, and it corresponds to an exponential extrapolation. We also consider linear extrapolation between normalized states in Supplemental Material [70] and we leave the discussion of other possible evolution paths to future works. Given the evolution via linear extrapolation, the time derivative equation of $|v(t)\rangle$ is $(d / d t)|v(t)\rangle=G|v(0)\rangle$, with $G=(\mathcal{M}-I) / T$. This corresponds to the case with $A(t)=G, B(t)=1$, and $\left|v^{\prime}(t)\right\rangle=|v(0)\rangle$ in Eq. (1), which can be variationally simulated.

For the second type, we assume that $\mathcal{M}$ is given as a tensor product of matrices, $\mathcal{M}=\mathcal{M}_{1} \otimes \cdots \otimes \mathcal{M}_{L}$, with each $\mathcal{M}_{i}$ acting on a small constant number of qubits. We can thus sequentially act $\mathcal{M}_{i}$ and focus on the realization of each term. For each $\mathcal{M} \equiv \mathcal{M}_{i}$, we first consider a singular value decomposition as $\mathcal{M}=U D V$, with unitary matrices $U, V$ and diagonal matrix $D$ with non-negative entries. Now we show how to realize the matrix multiplication of the three matrices. Given a spectral decomposition of $U=\sum_{j} e^{i \lambda_{j}}\left|\lambda_{j}\right\rangle\left\langle\lambda_{j}\right|$ with $\lambda_{j} \in \mathbb{R}$, we can represent it as $U=\exp \left(-i H^{U} T^{U}\right)$ with $H^{U}=-\sum_{j} \lambda_{j} / T^{U}\left|\lambda_{j}\right\rangle\left\langle\lambda_{j}\right|$ and 
$T^{U}>0$, which can be realized by evolving the state with Hamiltonian $H^{U}$ for time $T^{U}$ via variational real time simulation [13]. The realization is similar for $V$. To realize the diagonal matrix $D=\sum_{j} a_{j}|j\rangle\langle j|$, we approximate it as $D \approx \exp \left(-H^{D} T^{D}\right)$ with $-H^{D} T=$ $\sum_{a_{j} \neq 0} \log \left(a_{j}\right)|j\rangle\left\langle j\left|-\alpha \sum_{a_{j}=0}\right| j\right\rangle\langle j|$ and a constant $\alpha=$ $O\left[\log \left(1 / \varepsilon_{D}\right)+\log\right.$ Poly $\left.(n)\right]$ that ensures an accuracy of $\varepsilon_{D}$ of the approximation with $n$ qubits. We refer to Supplemental Material for a detailed discussion [70]. Therefore, we can define an unnormalized imaginary time evolution $[d|v(\tau)\rangle / d \tau]=-H^{D}|v(\tau)\rangle$, so that the initial vector $\left|v_{0}\right\rangle$ at $\tau=0$ is evolved to $D\left|v_{0}\right\rangle$ at $\tau=T$. Note that even though the second method assumes a tensor structure of $\mathcal{M}$, the matrix multiplication process can still be classically hard when the input state is a general multipartite entangled state. Such a case is practically relevant as we shortly discuss for its application in simulating jump processes of the stochastic Schrödinger equation.

Open system simulation.-Here we show quantum simulation of open system dynamics. Conventional algorithms for this task generally require a deep circuit [61-66]. The recently proposed variational approach [34] also needs two copies of the purified quantum state, thus requiring a number of qubits that is 4 times that of the system size. Our method, based on variational algorithms for generalized time evolution, matrix multiplication and the stochastic Schrödinger equation, instead only requires to apply shallow circuits on a single copy of the state without purification. Our algorithm thus extensively alleviates the requirement of quantum simulation of open systems.

Consider the Lindblad master equation,

$$
\frac{d}{d t} \rho=-i[H, \rho]+\mathcal{L} \rho,
$$

where $H$ describes the system Hamiltonian and $\mathcal{L} \rho=$ $\sum_{k} \frac{1}{2}\left(2 L_{k} \rho L_{k}^{\dagger}-L_{k}^{\dagger} L_{k} \rho-\rho L_{k}^{\dagger} L_{k}\right)$ describes the interaction with the environment with Lindblad operators $L_{k}$. Instead of simulating the evolution of the density matrix, we consider its equivalent description by the stochastic Schrödinger equation, which averages trajectories of pure state evolution under continuous measurements $[60,67]$. Each single trajectory $\left|\psi_{c}(t)\right\rangle$ is described by

$$
\begin{aligned}
d\left|\psi_{c}(t)\right\rangle= & \left(-i H-\frac{1}{2} \sum_{k}\left(L_{k}^{\dagger} L_{k}-\left\langle L_{k}^{\dagger} L_{k}\right\rangle\right)\right)\left|\psi_{c}(t)\right\rangle d t \\
& +\sum_{k}\left[\left(\frac{L_{k}\left|\psi_{c}(t)\right\rangle}{\| L_{k}\left|\psi_{c}(t)\right\rangle \|}-\left|\psi_{c}(t)\right\rangle\right) d N_{k}\right],
\end{aligned}
$$

where $d\left|\psi_{c}(t)\right\rangle=\left|\psi_{c}(t+d t)\right\rangle-\left|\psi_{c}(t)\right\rangle,\left\langle L_{k}^{\dagger} L_{k}\right\rangle=\left\langle\psi_{c}(t)\right.$ $\left.\left|L_{k}^{\dagger} L_{k}\right| \psi_{c}(t)\right\rangle$, and $d N_{k}$ randomly takes either 0 or 1, satisfying $d N_{k} d N_{k^{\prime}}=\delta_{k k^{\prime}} d N_{k}$ and $E\left[d N_{k}\right]=$ $\left\langle\psi_{c}(t)\left|L_{k}^{\dagger} L_{k}\right| \psi_{c}(t)\right\rangle d t$. At each time $t$, we can assume a positive-operator valued measure (POVM) measurement $\left\{O_{0}=I-\sum_{k} L_{k}^{\dagger} L_{k} d t, O_{k}=L_{k}^{\dagger} L_{k} d t\right\}$ happens. For measurement outcome $O_{k}$, the state discontinuously jumps to $L_{k}\left|\psi_{c}(t)\right\rangle / \| L_{k}\left|\psi_{c}(t)\right\rangle \|$ with probability $E\left[d N_{k}\right]$. And the total jump probability is $\gamma(t)=\sum_{k} E\left[d N_{k}\right]$. For outcome $O_{0}$ with probability $1-\gamma(t)$, we have $d N_{k}=0 \forall k$, and the state evolves under the generalized time evolution Eq. (1) with operator

$$
A=-i H-\frac{1}{2} \sum_{k}\left(L_{k}^{\dagger} L_{k}-\left\langle L_{k}^{\dagger} L_{k}\right\rangle\right)
$$

and $B(t)=1$. Here, $-i H$ corresponds to the conventional real time Schrödinger evolution with Hamiltonian $H$ and the other terms can be understood as a normalized damping process. Therefore, the whole process is composed of two parts: the continuous generalized time evolution and the quantum jump process.

The stochastic Schrödinger equation can be simulated with the Monto Carlo method. Suppose the state jumps at time $t$, then at time $t+\tau$, the probability $p(t+\tau)$ that the state does not jump is $p(t+\tau)=e^{-\Gamma(t, \tau)}$, with $\Gamma(t, \tau)=\int_{t}^{t+\tau} \gamma\left(t^{\prime}\right) d t^{\prime}$. When a jump happens at time $t$, a uniform random number $q \in[0,1]$ is generated. Then the time of the next jump is determined by accumulating time $\tau$ until we have $p(t+\tau)=q$. When a jump happens, a random number $q^{\prime} \in[0,1]$ is generated to determine which jump operator to apply at each time step. The state is updated to $L_{k}\left|\psi_{c}(t)\right\rangle / \| L_{k}\left|\psi_{c}(t)\right\rangle \|$ if $q^{\prime} \in\left[\tilde{\gamma}_{k-1}(t), \tilde{\gamma}_{k}(t)\right]$, where

$$
\tilde{\gamma}_{k}(t)=\frac{\sum_{l=1}^{k}\left\langle\psi_{c}(t)\left|L_{l}^{\dagger} L_{l}\right| \psi_{c}(t)\right\rangle}{\sum_{l=1}^{N_{L}}\left\langle\psi_{c}(t)\left|L_{l}^{\dagger} L_{l}\right| \psi_{c}(t)\right\rangle},
$$

and $N_{L}$ is the number of the Lindblad operators. Considering discretized time with initial state $\left|\psi_{c}(0)\right\rangle$, the stochastic Schrödinger equation from time 0 to $T$ can be simulated as follows.

Algorithm 1. Stochastic evolution equation.

1: Set $\Gamma=0$ and generate a random number $q \in[0,1]$.

2: for $t=0: d t: T d o$

3: $\quad$ if $e^{-\Gamma} \geq q$ then

4: $\quad$ Evolve the state $\left|\psi_{c}(t)\right\rangle$ under $A$ in Eq. (7).

5: $\quad$ Calculate $\gamma(t)=\sum_{k}\left\langle\psi_{c}(t)\left|L_{k}^{\dagger} L_{k}\right| \psi_{c}(t)\right\rangle d t$.

6: $\quad$ Update $\Gamma=\Gamma+\gamma(t)$.

7: else

8: $\quad$ Calculate $\tilde{\gamma}_{k}(t)$ in Eq. (8)

9: $\quad$ Generate a random number $q^{\prime} \in[0,1]$.

10: $\quad$ if $q^{\prime} \in\left[\tilde{\gamma}_{k-1}(t), \tilde{\gamma}_{k}(t)\right]$ then

11: $\quad$ Update $\left|\psi_{c}(t)\right\rangle$ to $L_{k}\left|\psi_{c}(t)\right\rangle / \| L_{k}\left|\psi_{c}(t)\right\rangle \|$.

12: $\quad \operatorname{Reset} \Gamma=0$ and randomly generate $q \in[0,1]$. 


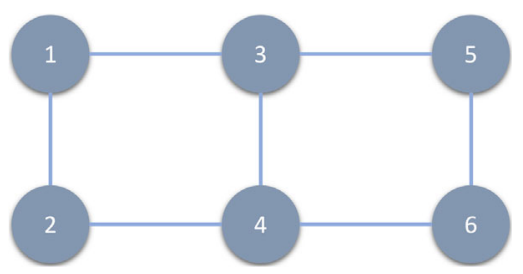

FIG. 1. Geometry of the 2D Ising model. We consider nearestneighbor interactions for two connected qubits.

Now we show how to variationally simulate the stochastic Schrödinger equation, algorithm 1. Suppose the state $\left|\psi_{c}(t)\right\rangle$ at time $t$ can be represented by the parametrized state $\left|\phi_{c}(\vec{\theta}(t))\right\rangle$ prepared by a quantum computer. We can simulate step 4, i.e., the evolution under operator $A$ defined in Eq. (7), with the algorithm for generalized time evolution. Specifically, we can evolve the parameters according to Eq. (3) with $\tilde{M}_{k, j}=\operatorname{Re}\left\{\left[\partial\langle\varphi(\vec{\theta}(t))| / \partial \theta_{k}\right]\left[\partial|\varphi(\vec{\theta}(t))\rangle / \partial \theta_{j}\right]\right\}$, $\tilde{V}_{k}=\operatorname{Re}\left\{\langle\varphi(\vec{\theta}(t))|[-i H-(L-\langle L\rangle)]\left[\partial|\varphi(\vec{\theta}(t))\rangle / \partial \theta_{k}\right]\right\}$, and $L=\frac{1}{2} \sum_{k} L_{k}^{\dagger} L_{k}$. The values of $\gamma(t)$ and $\tilde{\gamma}_{k}(t)$ at steps 5 and 8 are measured as expectation values of quantum states. The jump at step 11 is realized by the variational algorithms for matrix-vector multiplication. Especially, when considering $L_{k}$ as a product of operators of each qubits, it can be efficiently realized with the singular value decomposition method. In practice, we consider sparse Hamiltonian and Lindblad operators; therefore all the measurements can be efficiently evaluated. We refer to Supplemental Material for the discussion of the resource estimation [70].

Numerical simulation.-We numerically test the variational algorithm for simulating a 6-qubit 2D Ising model in a transverse field coupled to a Markovian bath [76-79]. The Hamiltonian is $H_{I}=J / 4 \sum_{\langle i j\rangle} Z_{i} Z_{j}+h_{X} \sum_{i=1}^{6} X_{i}$, where Pauli operators $X_{i}, Y_{i}$, and $Z_{i}$ act on the $i$ th spin and $\langle i j\rangle$ represents nearest-neighbor pairs in Fig. 1. The Lindblad term is $L_{i}=\sqrt{\gamma} \sigma_{i}^{+}$, with $\sigma_{i}^{+}=|1\rangle\left\langle\left. 0\right|_{i}\right.$ being the raising operator acting on the $i$ th spin. In our simulation, we set $J=1, h_{X}=1$, and $\gamma=1$. The initial state is prepared in a product state $|\varphi(0)\rangle=|0\rangle^{\otimes 6}$, and then the Hamiltonian $H_{I}$

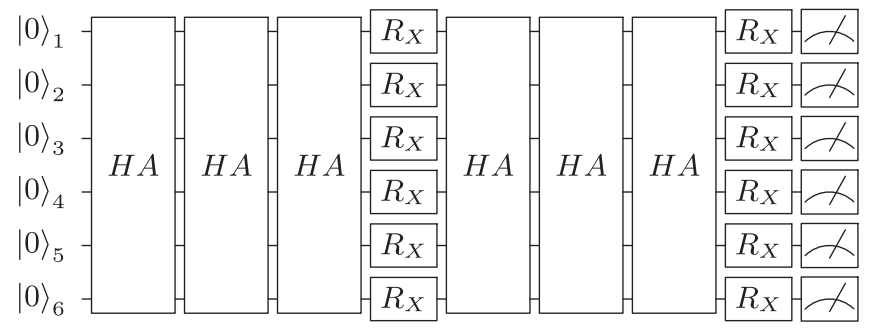

FIG. 2. The ansatz consists of the Hamiltonian ansatz (HA) and single qubit rotations. Each $\mathrm{HA}$ is $e^{\theta_{1}\left(Z_{5} Z_{6}\right)} e^{\theta_{2}\left(Z_{3} Z_{5}+Z_{4} Z_{6}\right)}$ $e^{\theta_{3}\left(Z_{3} Z_{4}\right)} e^{\theta_{4}\left(Z_{1} Z_{3}+Z_{2} Z_{4}\right)} e^{\theta_{5}\left(Z_{1} Z_{2}\right)} \quad e^{\theta_{6}\left(X_{3}+X_{4}\right)} e^{\theta_{7}\left(X_{1}+X_{2}+X_{5}+X_{6}\right)} \quad$ and each single qubit gate $R_{X}$ is $e^{-i \theta X}$. With different parameters for different HA and single qubit gates, the ansatz has in total 54 parameters. is suddenly turned on, which drives the qubits out of equilibrium. We simulate both the ideal and dissipative evolution from $t=0$ to $t=6$, and measure the normalized nearest-neighbor correlations $C=\sum_{\langle i j\rangle} Z_{i} Z_{j} / 7$. We use the Hamiltonian ansatz (HA) [80] sandwiched with single qubit rotations as the trial state, shown Fig. 2. The HA preserves the symmetry of the Hamiltonian and the single qubit rotations are introduced to break the symmetry, ensuring its capability for simulating the jump process.

We apply the algorithm in Ref. [13] and our algorithm 1 to simulate the ideal and dissipative evolution, respectively. We use the singular value decomposition method to simulate quantum jumps in algorithm 1 . We decompose the jump operator $\sigma^{+}$as $|1\rangle\langle 0|=| 1\rangle\langle 1| X \quad$ with $|1\rangle\langle 0|=U D V, U=I, D=| 1\rangle\langle 1|$, and $V=X$. To realize the $V$ operator, we set $H_{V}=X$ and $T_{V}=\pi / 2$ such that $X=\exp \left(-i H_{V} T_{V}\right)$. Then we evolve the state under Hamiltonian $H_{V}$ for time $T_{V}$ with time step $\delta t_{V}=0.01$ to have $X|\varphi(\vec{\theta})\rangle$. To realize $D=|1\rangle\langle 1|$, we set $H_{D}=|0\rangle\langle 0|$ and $T_{D}=6$ so that $D \approx \exp \left(-H_{D} T_{D}\right)$. Then we realize $D|\varphi(\vec{\theta})\rangle / \| D|\varphi(\vec{\theta})\rangle \|$ by using the normalized variational imaginary time evolution with total time $T_{D}$ and time step $\delta t_{D}=0.1$.

The simulation results are shown in Fig. 3. We compare the dynamical nearest-neighbor correlation of the simulation result and the exact evolution. We can see that the simulation result agrees well with exact evolution for both ideal and dissipative cases, thus verifying the functioning of variational quantum algorithms. The simulation result indicates a dissipation induced phase transition, where similar phenomena have been experimentally investigated in Ref. [81]. We expect our algorithm with medium-scale quantum hardware may be used for probing general interesting physics phenomena of many-body open systems.

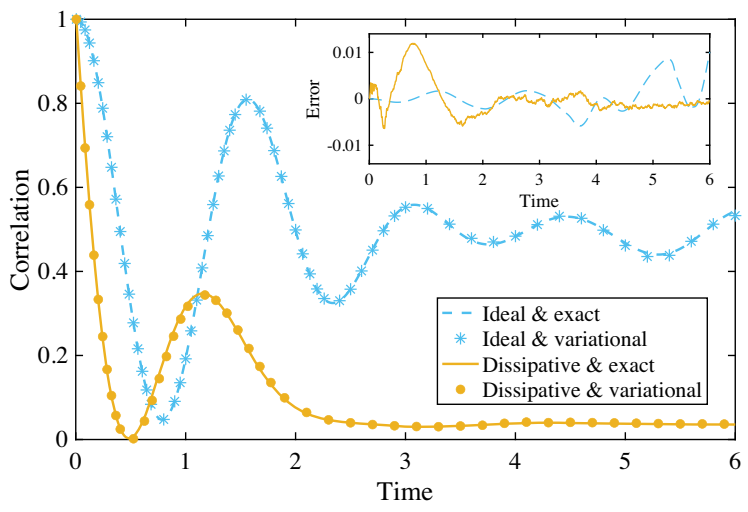

FIG. 3. Numerical simulation of ideal and dissipative evolution of the 2D Ising model. We consider evolution from $t=0$ to 6 with $\delta t=0.005$ and measure the nearest-neighbor correlations $C=\sum_{\langle i j\rangle} Z_{i} Z_{j} / 7$. We repeat the stochastic process of algorithm 1 with $N_{\text {trial }}=2 \times 10^{4}$ times. The results from variational simulation agree well with the exact ones, with a maximal error around 0.01 (see inset). 
Discussion.-To summarize, we extend the variational quantum simulation method to general processes, including generalized time evolution, and its application for solving linear algebra tasks and simulating the evolution of open quantum systems. Our algorithm for simulating the generalized time evolution can be applied to simulate non-Hermitian quantum mechanics [35-37] including nonequilibrium processes [38] and parity-time symmetric Hamiltonians [39-41]. Especially, it is shown in Ref. [41] that a quantum state can evolve to the target state faster with non-Hermitian parity-time symmetric Hamiltonians than the case with Hermitian Hamiltonians. Therefore, our variational algorithm for simulating the generalized time evolution may also be useful for designing faster quantum computing algorithms. Recently, several other algorithms for linear algebra tasks have been proposed to be compatible with near-term quantum hardware [82-85]. A thorough comparison of these algorithms and ours could also be an interesting future work. Our algorithm for simulating open systems only needs shallow circuits on a number of qubits matching the system size; thus it enables the possibility of investigating open system physics with near-term quantum computers. Finally, the proposed algorithms are compatible with NISQ hardware and can be further combined with quantum error mitigation techniques [13,17,86-92].

This work is supported by the EPSRC National Quantum Technology Hub in Networked Quantum Information Technology (EP/M013243/1). S. C. B. acknowledges support from the Europe Union AQTION project. We acknowledge the use of the University of Oxford Advanced Research Computing (ARC) facility in carrying out this work. S. E. is supported by Japan Student Services Organization (JASSO) Student Exchange Support Program (Graduate Scholarship for Degree Seeking Students). Y. L. is supported by National Natural Science Foundation of China (Grant No. 11875050) and NSAF (Grant No. U1930403). J. S. acknowledges support from China Scholarship Council (CSC). S. E. and J. S. acknowledge Shuxiang Cao for useful discussions.

*suguru.endou.uc@hco.ntt.co.jp

†xiao.yuan.ph@gmail.com

[1] R. Balian and M. Vénéroni, Ann. Phys. (N.Y.) 187, 29 (1988).

[2] F. Dalfovo, S. Giorgini, L. P. Pitaevskii, and S. Stringari, Rev. Mod. Phys. 71, 463 (1999).

[3] J. Haegeman, J. I. Cirac, T. J. Osborne, I. Pižorn, H. Verschelde, and F. Verstraete, Phys. Rev. Lett. 107, 070601 (2011).

[4] T. Shi, E. Demler, and J. I. Cirac, Ann. Phys. (Amsterdam) 390, 245 (2018).

[5] L. Vanderstraeten, J. Haegeman, and F. Verstraete, SciPost Phys. Lect. Notes 7, 21 (2019).

[6] E. Farhi, J. Goldstone, and S. Gutmann,arXiv:1411.4028.
[7] A. Peruzzo, J. McClean, P. Shadbolt, M.-H. Yung, Q. Zhou, P. J. Love, A. Aspuru-Guzik, and J. L. O'Brien, Nat. Commun. 5, 4213 (2014).

[8] Y. Wang, F. Dolde, J. Biamonte, R. Babbush, V. Bergholm, S. Yang, I. Jakobi, P. Neumann, A. Aspuru-Guzik, J. D. Whitfield et al., ACS Nano 9, 7769 (2015).

[9] P. J. J. O’Malley et al., Phys. Rev. X 6, 031007 (2016).

[10] Y. Shen, X. Zhang, S. Zhang, J.-N. Zhang, M.-H. Yung, and K. Kim, Phys. Rev. A 95, 020501(R) (2017).

[11] J. R. McClean, J. Romero, R. Babbush, and A. AspuruGuzik, New J. Phys. 18, 023023 (2016).

[12] S. Paesani, A. A. Gentile, R. Santagati, J. Wang, N. Wiebe, D. P. Tew, J. L. O'Brien, and M. G. Thompson, Phys. Rev. Lett. 118, 100503 (2017).

[13] Y. Li and S. C. Benjamin, Phys. Rev. X 7, 021050 (2017).

[14] J. I. Colless, V. V. Ramasesh, D. Dahlen, M. S. Blok, M. E. Kimchi-Schwartz, J. R. McClean, J. Carter, W. A. de Jong, and I. Siddiqi, Phys. Rev. X 8, 011021 (2018).

[15] R. Santagati, J. Wang, A. A. Gentile, S. Paesani, N. Wiebe, J. R. McClean, S. Morley-Short, P. J. Shadbolt, D. Bonneau, J. W. Silverstone, D. P. Tew, X. Zhou, J. L. O'Brien, and M. G. Thompson, Sci. Adv. 4, eaap9646 (2018).

[16] A. Kandala, A. Mezzacapo, K. Temme, M. Takita, M. Brink, J. M. Chow, and J. M. Gambetta, Nature (London) 549, 242 (2017).

[17] A. Kandala, K. Temme, A. D. Córcoles, A. Mezzacapo, J. M. Chow, and J. M. Gambetta, Nature (London) 567, 491 (2019).

[18] C. Hempel, C. Maier, J. Romero, J. McClean, T. Monz, H. Shen, P. Jurcevic, B. P. Lanyon, P. Love, R. Babbush, A. Aspuru-Guzik, R. Blatt, and C. F. Roos, Phys. Rev. X 8, 031022 (2018).

[19] J. Romero, R. Babbush, J. R. McClean, C. Hempel, P. J. Love, and A. Aspuru-Guzik, Quantum Sci. Technol. 4, 014008 (2018).

[20] S. McArdle, T. Jones, S. Endo, Y. Li, S. C. Benjamin, and X. Yuan, npj Quantum Inf. 5, 75 (2019).

[21] T. Jones, S. Endo, S. McArdle, X. Yuan, and S. C. Benjamin, Phys. Rev. A 99, 062304 (2019).

[22] O. Higgott, D. Wang, and S. Brierley, Quantum 3, 156 (2019).

[23] R. Santagati, J. Wang, A. A. Gentile, S. Paesani, N. Wiebe, J. R. McClean, S. Morley-Short, P. J. Shadbolt, D. Bonneau, J. W. Silverstone et al., Sci. Adv. 4, eaap9646 (2018).

[24] J. R. McClean, M. E. Kimchi-Schwartz, J. Carter, and W. A. de Jong, Phys. Rev. A 95, 042308 (2017).

[25] J. I. Colless, V. V. Ramasesh, D. Dahlen, M. S. Blok, M. E. Kimchi-Schwartz, J. R. McClean, J. Carter, W. A. de Jong, and I. Siddiqi, Phys. Rev. X 8, 011021 (2018).

[26] C. Kokail, C. Maier, R. van Bijnen, T. Brydges, M. K. Joshi, P. Jurcevic, C. A. Muschik, P. Silvi, R. Blatt, C. F. Roos et al., Nature (London) 569, 355 (2019).

[27] I. Kassal, J. D. Whitfield, A. Perdomo-Ortiz, M.-H. Yung, and A. Aspuru-Guzik, Annu. Rev. Phys. Chem. 62, 185 (2011).

[28] D. Lu, B. Xu, N. Xu, Z. Li, H. Chen, X. Peng, R. Xu, and J. Du, Phys. Chem. Chem. Phys. 14, 9411 (2012).

[29] S. Kais, Quantum Information and Computation for Chemistry (Wiley, Hoboken, 2014), https://onlinelibrary.wiley .com/doi/book/10.1002/9781118742631. 
[30] S. McArdle, S. Endo, A. Aspuru-Guzik, S. C. Benjamin, and X. Yuan, Rev. Mod. Phys. 92, 015003 (2020).

[31] J. Preskill, Quantum 2, 79 (2018).

[32] K. M. Nakanishi, K. Mitarai, and K. Fujii, Phys. Rev. Research 1, 033062 (2019).

[33] K. Heya, K. M. Nakanishi, K. Mitarai, and K. Fujii, arXiv:1904.08566.

[34] X. Yuan, S. Endo, Q. Zhao, Y. Li, and S. C. Benjamin, Quantum 3, 191 (2019).

[35] N. Hatano and D. R. Nelson, Phys. Rev. Lett. 77, 570 (1996).

[36] N. Moiseyev, Non-Hermitian Quantum Mechanics (Cambridge University Press, Cambridge, England, 2011).

[37] F. Bagarello, J.-P. Gazeau, F. H. Szafraniec, and M. Znojil, Non-Selfadjoint Operators in Quantum Physics: Mathematical Aspects (John Wiley \& Sons, Hoboken, 2015).

[38] H. C. Fogedby, A. B. Eriksson, and L. V. Mikheev, Phys. Rev. Lett. 75, 1883 (1995).

[39] C. M. Bender and S. Boettcher, Phys. Rev. Lett. 80, 5243 (1998).

[40] C. M. Bender, Rep. Prog. Phys. 70, 947 (2007).

[41] C. M. Bender, D. C. Brody, H. F. Jones, and B. K. Meister, Phys. Rev. Lett. 98, 040403 (2007).

[42] I. Rotter, J. Phys. A 42, 153001 (2009).

[43] C. E. Rasmussen, Advanced Lectures on Machine Learning (Springer, New York, 2004), pp. 63-71.

[44] N. M. Nasrabadi, J. Electron. Imaging 16, 049901 (2007).

[45] A. W. Harrow, A. Hassidim, and S. Lloyd, Phys. Rev. Lett. 103, 150502 (2009).

[46] A. Childs, R. Kothari, and R. Somma, SIAM J. Comput. 46, 1920 (2017).

[47] A. Ambainis, in Proceedings of the 29th Symposium on Theoretical Aspects of Computer Science, STACS'12 (LIPIcs, Wadern, 2012), Vol. 14, pp. 636-647.

[48] B. D. Clader, B. C. Jacobs, and C. R. Sprouse, Phys. Rev. Lett. 110, 250504 (2013).

[49] L. Wossnig, Z. Zhao, and A. Prakash, Phys. Rev. Lett. 120, 050502 (2018).

[50] S. Chakraborty, A. Gilyén, and S. Jeffery, in Proceedings of the 46th International Colloquium on Automata, Languages, and Programming (ICALP 2019), Leibniz International Proceedings in Informatics (LIPIcs) Vol. 132, edited by C. Baier, I. Chatzigiannakis, P. Flocchini, and S. Leonardi (Schloss Dagstuhl-Leibniz-Zentrum fuer Informatik, Dagstuhl, Germany, 2019), pp. 33:1-33:14.

[51] A. Gilyén, Y. Su, G. H. Low, and N. Wiebe, in Proceedings of the 51st Annual ACM SIGACT Symposium on Theory of Computing (Association for Computing Machinery, New York, 2019), pp. 193-204, https://doi.org/10.1145/ 3313276.3316366.

[52] Y. Subaş1, R. D. Somma, and D. Orsucci, Phys. Rev. Lett. 122, 060504 (2019).

[53] P. Rebentrost, M. Mohseni, and S. Lloyd, Phys. Rev. Lett. 113, 130503 (2014).

[54] S. Lloyd, M. Mohseni, and P. Rebentrost, Nat. Phys. 10, 631 (2014).

[55] J. Biamonte, P. Wittek, N. Pancotti, P. Rebentrost, N. Wiebe, and S. Lloyd, Nature (London) 549, 195 (2017).

[56] C. Shao, arXiv:1803.01601.

[57] K. Mitarai, M. Negoro, M. Kitagawa, and K. Fujii, Phys. Rev. A 98, 032309 (2018).
[58] G. Lindblad, Commun. Math. Phys. 48, 119 (1976).

[59] H.-P. Breuer, F. Petruccione et al., The Theory of Open Quantum Systems (Oxford University Press on Demand, Oxford, 2002), ISBN 9780198520634.

[60] C. Gardiner and P. Zoller, Quantum Noise: A Handbook of Markovian and Non-Markovian Stochastic Process with Applications to Quantum Optics (2000).

[61] H. Wang, S. Ashhab, and F. Nori, Phys. Rev. A 83, 062317 (2011).

[62] D. Bacon, A. M. Childs, I. L. Chuang, J. Kempe, D. W. Leung, and X. Zhou, Phys. Rev. A 64, 062302 (2001).

[63] R. Sweke, I. Sinayskiy, D. Bernard, and F. Petruccione, Phys. Rev. A 91, 062308 (2015).

[64] R. Sweke, M. Sanz, I. Sinayskiy, F. Petruccione, and E. Solano, Phys. Rev. A 94, 022317 (2016).

[65] R. Cleve and C. Wang, arXiv:1612.09512.

[66] A. M. Childs and T. Li, Quantum Inf. Comput. 17, 901 (2017).

[67] H. J. Carmichael, Phys. Rev. Lett. 70, 2273 (1993).

[68] A. McLachlan, Mol. Phys. 8, 39 (1964).

[69] K. Mitarai and K. Fujii, Phys. Rev. Research 1, 013006 (2019).

[70] See Supplemental Material at http://link.aps.org/ supplemental/10.1103/PhysRevLett.125.010501 for resource estimations and detailed derivations.

[71] S. Lloyd, Science 273, 1073 (1996).

[72] M. Suzuki, J. Math. Phys. (N.Y.) 32, 400 (1991).

[73] A. M. Childs, A. Ostrander, and Y. Su, Quantum 3, 182 (2019).

[74] D. W. Berry, A. M. Childs, R. Cleve, R. Kothari, and R. D. Somma, Phys. Rev. Lett. 114, 090502 (2015).

[75] G. H. Low and I. L. Chuang, Quantum 3, 163 (2019).

[76] T. E. Lee, H. Haffner, and M. C. Cross, Phys. Rev. Lett. 108, 023602 (2012).

[77] C. Ates, B. Olmos, J. P. Garrahan, and I. Lesanovsky, Phys. Rev. A 85, 043620 (2012).

[78] I. Lesanovsky, M. van Horssen, M. Guţă, and J. P. Garrahan, Phys. Rev. Lett. 110, 150401 (2013).

[79] D. C. Rose, K. Macieszczak, I. Lesanovsky, and J. P. Garrahan, Phys. Rev. E 94, 052132 (2016).

[80] D. Wecker, M. B. Hastings, and M. Troyer, Phys. Rev. A 92 , 042303 (2015).

[81] J. Raftery, D. Sadri, S. Schmidt, H. E. Türeci, and A. A. Houck, Phys. Rev. X 4, 031043 (2014).

[82] X. Xu, J. Sun, S. Endo, Y. Li, S. C. Benjamin, and X. Yuan, arXiv:1909.03898.

[83] C. Bravo-Prieto, R. LaRose, M. Cerezo, Y. Subasi, L. Cincio, and P. J. Coles, arXiv:1909.05820.

[84] D. An and L. Lin, arXiv:1909.05500.

[85] H.-Y. Huang, K. Bharti, and P. Rebentrost, arXiv:1909.07344.

[86] S. McArdle, X. Yuan, and S. Benjamin, Phys. Rev. Lett. 122, 180501 (2019).

[87] X. Bonet-Monroig, R. Sagastizabal, M. Singh, and T. E. O’Brien, Phys. Rev. A 98, 062339 (2018).

[88] K. Temme, S. Bravyi, and J. M. Gambetta, Phys. Rev. Lett. 119, 180509 (2017).

[89] S. Endo, S. C. Benjamin, and Y. Li, Phys. Rev. X 8, 031027 (2018).

[90] M. Huo and Y. Li, arXiv:1811.02734.

[91] M. Otten and S. K. Gray, npj Quantum Inf. 5, 11 (2019).

[92] J. Sun, X. Yuan, T. Tsunoda, V. Vedral, S. C. Bejamin, and S. Endo, arXiv:2001.04891. 